\title{
Terapia miofuncional, maloclusión y disfunción orofacial: Evaluación fotogramétrica del perfil.
}

\section{Myofunctional therapy, malocclusion and orofacial dysfunction: Profile photogrammetric evaluation.}

\author{
Alicia Muñoz-Díaz ${ }^{1}$, Lorena Sepúlveda-Vega ${ }^{2}$, Oscar Norambuena-Lama ${ }^{1}$, \\ Rocio Serrano-Herrera ${ }^{1}$, Cristián Vergara-Núñez ${ }^{2^{*}}$
}

1.Práctica Privada, Santiago, Chile. 2.Departamento del Niño y Ortopedia Dentomaxilar, Facultad de Odontología, Universidad de Chile, Santiago, Chile.

${ }^{*}$ Correspondencia Autor: Cristian Marcelo Vergara Núñez | Dirección: Olivos 943, Independencia, Región Metropolitana. Departamento del Niño y Ortopedia Dentomaxilar, Facultad de Odontología, Universidad de Chile, Santiago, Chile. | E-mail: crvergar@uchile.cl| Código Postal: 8380000. Trabajo recibido el 27/08/2020

Trabajo revisado 08/02/2021

Aprobado para su publicación el 06/03/2021

\begin{abstract}
RESUMEN
El objetivo de este estudio observacional analítico es determinar el efecto de la Terapia Miofuncional en el perfil facial de voluntarios con maloclusión y disfunción orofacial, mediante fotogrametría. En una muestra de 15 pacientes, se midieron indirectamente mediante fotogrametría en el Software Blue Sky Plan ${ }^{\circledR}$ las siguientes variables cefalométricas: Ángulo Nasolabial, Prominencia Labial y Plano Estético. Se realizó toma de fotografías al comenzar la Terapia Miofuncional (primera sesión) y al finalizarla (décima sesión). Para comparar valores obtenidos en el mismo voluntario en cada variable cefalométrica entre principio y final de terapia, análisis estadísticos fueron realizados. Se determinó que no hay diferencia estadística en valores obtenidos en ninguna de las variables cefalométricas al comparar el inicio con el final de terapia. Por consiguiente, se concluyó que la Terapia Miofuncional no tiene un efecto significativo en estas variables de perfil facial en voluntarios con maloclusión y disfunción orofacial medidas mediante fotogrametría, pese a cambios funcionales percibidos por pacientes y evaluadores. Es fundamental poder realizar un diagnóstico riguroso y posterior derivación a Fonoaudiología, si corresponde, para lograr equilibrio funcional en los pacientes y reducir riesgo de progresión o reaparición de Anomalías Dentomaxilares.

PALABRAS CLAVE

Fotogrametría; Perfil facial; Terapia miofuncional.
\end{abstract}

Int. J. Inter. Dent Vol. 14(3); 229-232, 2021.

\section{ABSTRACT}

This analytical observational study aimed to determine the effect of Myofunctional Therapy on the facial profile in volunteers with malocclusion and orofacial dysfunction, through photogrammetry. Fifteen patients were recruited, and the following cephalometric variables were measured indirectly through photogrammetry in the Blue Sky Plan Software (Blue Sky $\mathrm{Bio}^{\circledR}$ ): Nasolabial Angle, Lip Prominence and Aesthetic Plane. Previous photographs were taken, at the beginning of Myofunctional Therapy (first session) and the end (tenth session). To compare the values of each variable in a volunteer between the beginning and end of therapy, statistical analyses were performed. There was no statistical difference in the values obtained in any of these variables when comparing the beginning and the end of myofunctional therapy. Therefore, it was concluded that myofunctional therapy does not have a statistically significant effect on these facial profile variables in volunteers with malocclusion and orofacial dysfunction measured by photogrammetry, despite the functional changes perceived by patients and evaluators. However, it is essential to perform a rigorous diagnosis and subsequent referral to speech therapy, if applicable, to achieve functional balance in the patients and reduce progression or recurrence risk of Dentomaxillary Abnormalities.

\section{KEY WORDS}

Photogrammetry; Facial profile; Myofunctional therapy.

Int. J. Inter. Dent Vol. 14(3); 229-232, 2021.

\section{INTRODUCCIÓN}

Algunas maloclusiones están relacionadas con alteraciones en las funciones orofaciales ${ }^{(1,2,3)}$. Estos hábitos no funcionales alteran la posición normal de los dientes, en la postura del sujeto y también pueden observarse en sus tejidos blandos ${ }^{(4)}$ (Figura 1). Por este motivo es necesaria la colaboración entre Ortodoncia y Fonoaudiología.
De acuerdo con la literatura y la experiencia clínica, se ha propuesto que la Terapia Miofuncional (TM) podría ejercer cambios cuantificables en los tejidos blandos que se verán reflejados en el análisis cefalométrico, empleando la fotogrametría facial $\left.\right|^{(5,6,7)}$. El objetivo de este estudio es determinar el efecto de la TM en el perfil facial en pacientes con maloclusión y disfunción orofacial mediante el uso de fotogrametría. 


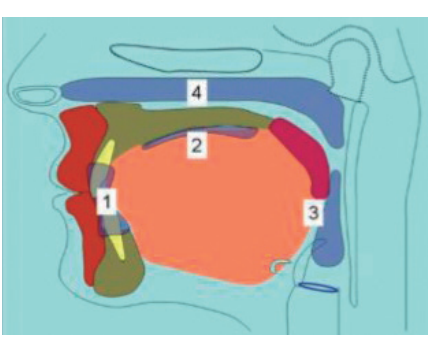

Figura 1. Modelo de compartimentos biofuncionales del sistema orofacial: Compartimiento Inter-Oclusal(1), Compartimiento sub-palatino(2). La vía aérea superior, junto con el esfínter velofaríngeo como límites, forman otros dos compartimentos biofuncionales ${ }^{(3,4)}$. Modificado de Engelke et $\mathrm{al}^{(4)}$.

\section{MATERIALES Y MÉTODOS:}

Muestra: Veinte $(n=20)$ voluntarios fueron seleccionados mediante método por conveniencia dentro de los pacientes de las Clínicas de Odontopediatría Básica e Integral y UTE del Niño y Adolescente III y IV, dependientes del Departamento del Niño y Ortopedia Dentomaxilar de la Facultad de Odontología de la Universidad de Chile, derivados a terapia fonoaudiológica en la Clínica Odontológica de la Facultad de Odontología de la Universidad de Chile (FOUCH) en los años 2017 y 2018. Finalmente, debido a los criterios de exclusión, la muestra correspondió a quince $(n=15)$ voluntarios obtenidos de la muestra inicial.

A los participantes mayores de edad (pacientes con 18 años) se les explicó en forma verbal y escrita, a través del proceso de Consentimiento Informado, los objetivos de la investigación, el procedimiento en que consistiría, sus riesgos y el manejo de los datos. Al aceptar la participación en el estudio se pidió que firmaran el documento de Consentimiento Informado.

A los participantes menores de edad se les explicó en forma verbal y escrita, junto a sus tutores, a través del proceso de Asentimiento Informado, los objetivos de la investigación, el procedimiento en que consistiría, sus riesgos y el manejo de los datos. Al aceptar la participación en el estudio se pidió a ellos y sus tutores que firmaran el documento de Asentimiento Informado.

Los criterios de inclusión del grupo fueron: Mujeres y Hombres. Entre 5 y 18 años. Los criterios de exclusión del grupo fueron: Voluntarios en situación de discapacidad cognitiva y/o psicomotora (que les impida mantener una postura estable o no sean capaces de seguir instrucciones). Pacientes en tratamiento de ortodoncia. Pacientes que no asistieron a más de una sesión habiendo confirmado previamente. Pacientes que abandonaron el tratamiento $\mathrm{y}$, por ende, no completaron las sesiones de la terapia fonoaudiológica.

Una vez que el participante (o su tutor, según corresponda) firmó el consentimiento o asentimiento informado, fue citado para llevar a cabo la toma de fotografías previo a comenzar la terapia fonoaudiológica y una vez finalizada (el mismo día que fueron citados a terapia fonoaudiológica). El set fotográfico fue montado en la Clínica Odontológica de la FOUCH. Las fotografías fueron tomadas por investigadores previamente calibrados y capacitados del Área de Ortodoncia de la $\mathrm{FOUCH}$, acompañados por un ortodoncista experto. Los voluntarios derivados a terapia fonoaudiológica fueron atendidos por la Fonoaudióloga de la FOUCH con previa citación agendada y confirmada telefónicamente.

\section{Procedimiento}

\section{Descripción del Tratamiento Miofuncional}

La TM aplicada en este estudio, fue el protocolo de terapia para tratamiento de respiración oral adaptado por Prof. Flga. Lorena Sepúlveda, Facultad de Odontología, Universidad de Chile, según su experticia, a partir de Korb( ${ }^{(8)}$, Universidad de Sao Paulo (Tabla 1).

\section{Descripción de la toma fotográfica}

El protocolo fotográfico que se utilizó en este trabajo fue el de Astudillo et $\mathrm{al}^{(9)}$, que consistió en utilizar una cámara réflex digital (Pentax ${ }^{\circledR}$, modelo K-3), con una resolución de 24 megapíxeles, con un lente fijo de distancia focal $100 \mathrm{~mm}$ (modelo Asahi SMC f4.0 Macro, Pentax ${ }^{\circledR}$ ) y encuadrada a $30 \mathrm{~cm}$ lo que da una distancia sujeto-cámara de $205 \mathrm{~cm}$.

\section{Parámetros de la cámara fotográfica}

La cámara fue soportada por un trípode (modelo GT1544T, Gitzo ${ }^{\circledR}$ ) con un nivel de burbuja doble integrado, posicionado a la altura de la cabeza del voluntario, puesto de perfil, específicamente con el lente apuntando a la altura del ala de la nariz.
Tabla 1: Protocolo de terapia para tratamiento de respiración oral adaptado por Prof. Flga. Lorena Sepúlveda, Facultad de Odontología, Universidad de Chile a partir de Korb ${ }^{(8)}$, Universidad de Sao Paulo

\begin{tabular}{|c|c|}
\hline Objetivos de la terapıa Mıofuncional & Medios para alcanzar los objetivos \\
\hline \multirow{3}{*}{$\begin{array}{l}\text { 1. Reorganizar la postura corporal } \\
\text { general }\end{array}$} & $\begin{array}{l}\begin{array}{l}\text { Realización de ejercicio para elongar } \\
\text { musculatura cervical }\end{array} \\
\end{array}$ \\
\hline & $\begin{array}{l}\text { Realización de ejercicio para elongar } \\
\text { musculatura lateral de columna }\end{array}$ \\
\hline & Concientización y modificación de postura corporal \\
\hline \multirow{2}{*}{$\begin{array}{l}\text { 2. Concientizar respecto a la función } \\
\text { respiratoria }\end{array}$} & Percepción del patrón respiratorio realizado \\
\hline & $\begin{array}{l}\begin{array}{l}\text { Concientización so } \\
\text { normal/adecuado }\end{array} \\
\end{array}$ \\
\hline 3. Realizar limpieza nasal & Aplicación de suero fisiológico en narinas \\
\hline 4. Promover uso de vía respiratoria nasal & \begin{tabular}{|lrlll} 
Realización de ejercicios de inspiración y \\
espiración nasal
\end{tabular} \\
\hline \multirow{2}{*}{$\begin{array}{l}\text { 5. Reposicionar la lengua en posición de } \\
\text { reposo } \\
6 \text {. Fortalecer los músculos: orbicular de la } \\
\text { boca, buccinador, elevadores de la } \\
\text { mandíbula, músculos extrínsecos e } \\
\text { intrínseco de la lengua }\end{array}$} & $\begin{array}{l}\text { Maniobras para generar presión intraoral y } \\
\text { compartimentos biofuncionales }\end{array}$ \\
\hline & niofuncionales \\
\hline $\begin{array}{l}\text { 7.Establecer la vía respiratoria nasal en } \\
\text { reposo }\end{array}$ & asal \\
\hline $\begin{array}{l}\text { 8.Promover tipo respiratorio medio } \\
\text { inferior }\end{array}$ & $\begin{array}{l}\text { Realización de entrenamiento respir } \\
\text { neumofonoarticulatorio implicando la en } \\
\text { fonemas }\end{array}$ \\
\hline $\begin{array}{l}\text { 9. Establecer conductas correctas en los } \\
\text { patrones deglutorios y en la coordinación } \\
\text { entre las funciones de respiración } \\
\text { deglución }\end{array}$ & $\begin{array}{l}\text { Manejo de diferentes consistencias en deglución y } \\
\text { corrección de patrones deglutorios erróneos }\end{array}$ \\
\hline \multirow{2}{*}{$\begin{array}{l}\text { 10.Establecer un correcto patrón } \\
\text { masticatorio } \\
\text { 11.Promover la respiración nasal durante } \\
\text { la masticación }\end{array}$} & $\begin{array}{l}\text { nancia bilateral en la } \\
\text { nsistencias }\end{array}$ \\
\hline & $\begin{array}{l}\text { Realización de entrenamiento respiratorio durante } \\
\text { la masticación }\end{array}$ \\
\hline $\begin{array}{l}\text { 12. Promover la coordinación entre las } \\
\text { funciones de respiración y deglución } \\
13 \text { Establecer puntos y modos }\end{array}$ & $\begin{array}{l}\text { Realización de entrenamiento respiratorio durante } \\
\text { la deglución de alimento sólidos y líquidos }\end{array}$ \\
\hline 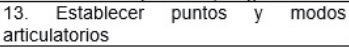 & $\begin{array}{l}\text { Corregir puntos y modos articulatorios que } \\
\text { presenten impresiones }\end{array}$ \\
\hline $\begin{array}{l}\text { 14. Promover la coordinación } \\
\text { fonorrespiratoria }\end{array}$ & $\begin{array}{l}\text { Realización de entrenamiento de coordinación } \\
\text { neumofonoarticulatoria involucrando la lectura de } \\
\text { frases de textos. }\end{array}$ \\
\hline $\begin{array}{l}\text { 15. Promover patrones de mímica facial } \\
\text { que favorecen el desarrollo del sistema } \\
\text { estomatognático }\end{array}$ & $\begin{array}{l}\text { Realización de ejercicios de mímica facial } \\
\text { pertinentes a cada contexto de uso y comunicativo }\end{array}$ \\
\hline \multicolumn{2}{|c|}{$\begin{array}{l}\text { - Los ejercicios y objetivos de tratamiento con cada paciente fueron escogidos según la } \\
\text { necesidad de cada uno. }\end{array}$} \\
\hline \multicolumn{2}{|c|}{$\begin{array}{l}\text { - Se realizaron } 10 \text { sesiones con cada paciente, en frecuencia de } 1 \text { vez por semana por } \\
\text { periodos de } 30 \text { minutos. }\end{array}$} \\
\hline
\end{tabular}

La cámara fue usada en modo manual, con los siguientes parámetros: ISO-100; f/11; Velocidad de obturación $1 / 125$ seg. Además, se utilizó un flash externo (modelo 540 FGZ II, Pentax ${ }^{\circledR}$ ) programado en manual con difusor para mejorar la iluminación.

\section{Parámetros del voluntario}

Se fotografió a cada paciente al inicio del tratamiento y al final (décima sesión). Cada sesión tuvo una duración aproximada de 30 minutos, con intervalos entre sesiones según disponibilidad de la profesional, dando prioridad a los voluntarios de este estudio. Se solicitó a cada voluntario que retirara objetos que pudieran interferir con las mediciones, tales como audífonos, aros, lentes u otros. También se les solicitó que se quiten el calzado. Fue necesario ocultar el pelo detrás del pabellón auricular, para facilitar la visualización de éste.

Para alcanzar una Posición Natural de Cabeza (PNC)(10) se utilizó el protocolo propuesto por Solow y Tallgren ${ }^{(11)}$ que consiste en pedirle al voluntario que realice una pequeña caminata previo a su ingreso al set fotográfico. Una vez que ingresa, éste realiza una marcha breve sin desplazarse; luego realiza movimientos de flexión y extensión de cabeza con una amplitud decreciente hasta alcanzar un balance natural y se le pide al sujeto que mire hacia el horizonte. Se solicita que trague saliva y se espera dos o tres segundos, para permitir obtener una posición de reposo mandibular para finalmente realizar la toma fotográfica (Figura 2).

\section{Protocolo para la obtención de la imagen}

El trípode fue ajustado para igualar la altura de la cámara al ala de la nariz del voluntario. Para lograr la correcta inclinación de la toma fotográfica se utilizó la función de giroscopio electrónico integrada en la cámara utilizada, lo que permitió obtener una imagen orientada en una horizontal y vertical verdadera.

Una vez que fueron cumplidos los parámetros de la cámara fotográfica y parámetros del voluntario, se procedió a la toma fotográfica.

\section{Definición de medidas utilizadas}

Se realizó la evaluación estética propuesta por Legan y Burstone ${ }^{(12)}$, y Ricketts ${ }^{(13)}$ (Figura 3).

Ángulo Nasolabial (Legan) (Figura 3a): ángulo formado entre los puntos Ls-Sn-Cm.

Interpretación: Sirve para evaluar la protrusión labial, pero puede estar influenciado por la columela. Este ángulo es muy influenciable 


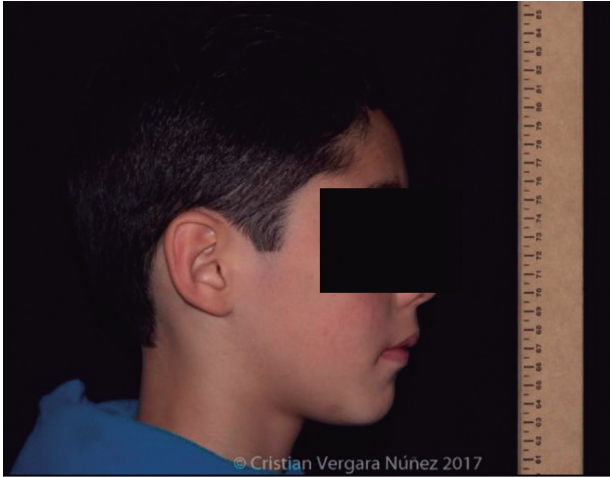

Figura 2. Ejemplo de fotografía de voluntario en Posición Natural de Cabeza.

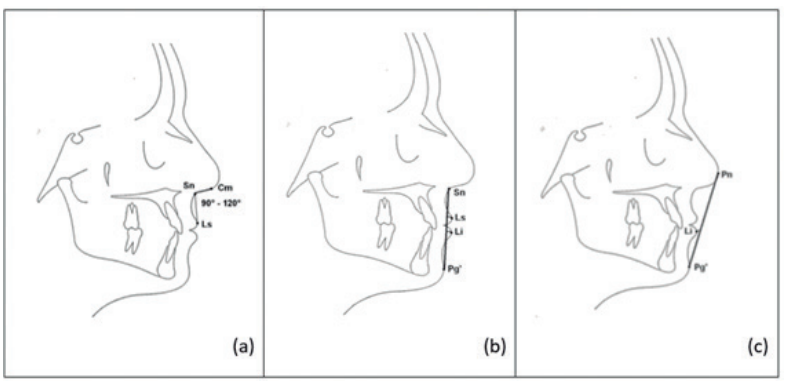

Figura 3. Evaluación estética propuesta en Legan y Burstone12, y Ricketts13. (a) Ángulo Nasolabial (Legan). (b) Prominencia Labial (Burstone). (c) Plano Estético (Ricketts).

por los tratamientos ortodóncicos que modifican la posición del diente incisivo superior y debe siempre tomarse en cuenta.

Prominencia Labial (Burstone) (Figura 3b): Se midió desde el labio superior y labio inferior a la línea Sn-Pg'.

Interpretación: Estas dos medidas sirven para evaluar la posición de los labios en sentido anteroposterior. Valores mayores a la norma indican protrusión y menores a la norma clínica, retrusión del labio.

Plano Estético (Ricketts) (Figura 3c): Se midió desde Pn a Pg'.

Interpretación: Describe la armonía de los labios en relación con la nariz y el mentón. Los labios se ubican por delante o por detrás de esta línea dependiendo de la edad. El labio inferior está ligeramente por delante del labio superior.

\section{Análisis de los datos}

\section{Mediciones}

Se procedió a la medición de las medidas lineales y angulares en la evaluación cefalométrica y se calibró respecto a la regla utilizada para medir el encuadre en el set fotográfico mediante el uso del programa Blue Sky Plan (Blue Sky Bio ${ }^{\circledR}$ ).

\section{Análisis estadístico}

Las 4 mediciones cefalométricas ( 1 angular, 3 lineales) fueron medidas en cada uno de los voluntarios al inicio y al final del tratamiento. Esto obtuvo un total de 120 mediciones. Todos los datos fueron tabulados en una planilla Excel (Microsoft Excel ${ }^{\circledR} 2010$ ) y se procesaron estadísticamente con el Software Stata $14 \mathrm{~S} \mathrm{E}^{\circledR}$ (de Stata Corporation L.P.).

\section{RESULTADOS}

Para cada una de las cuatro variables cefalométricas: Ángulo Nasolabial, Prominencia Labial: Labio superior y Labio Inferior y Plano Estético: Labio inferior, se realizaron mediciones al inicio del tratamiento (T1) y al final de este (T2), obteniéndose un total de 120 registros, que se utilizaron para los análisis estadísticos.

Para analizar el tipo de distribución de la muestra, se realizó el test de Shapiro Wilk, que demostró una distribución normal $(p>0,05)$.

Luego, se aplicó un T-Test Pareado para determinar si existía diferencia estadística en cada variable entre T1 y T2, con un intervalo de confianza del 95\%. Los valores obtenidos se presentan en la Tabla 2.

A partir de las pruebas realizadas, se observó que no existieron diferencias estadísticas entre las mediciones en T1 y T2 para las variables cefalométricas estudiadas en este grupo de pacientes.

Tabla 2: Resumen de resultados obtenidos luego de las mediciones y sus respectivas pruebas estadísticas. T1: inicio del tratamiento. T2: final del tratamiento. $\left(^{*}\right)$ medido en grados. $\left(^{* *}\right)$ medido en milímetros.

\begin{tabular}{|c|c|c|c|c|c|c|c|c|c|c|c|}
\hline \multirow{2}{*}{ Medición } & \multicolumn{2}{|c|}{ PROMEDIO } & \multirow{2}{*}{\multicolumn{2}{|c|}{\begin{tabular}{|c|c|} 
DESV. EST. \\
T1 & $\mathrm{T} 2$ \\
\end{tabular}}} & \multicolumn{2}{|c|}{ MIN } & \multicolumn{2}{|c|}{$\operatorname{MAX}$} & \multicolumn{2}{|c|}{ TEST DE SHAPIRO-WILK } & \multirow{2}{*}{$\begin{array}{c}\text { T-TEST } \\
\text { T1-T2 }\end{array}$} \\
\hline & 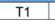 & T2 & & & & $\mathrm{T} 2$ & $\mathrm{~T} 1$ & $\mathrm{~T} 2$ & $\mathrm{~T} 1$ & $\mathrm{~T} 2$ & \\
\hline Angulo nasolabial (") & 107,31 & 109,54 & 11,94 & 9,89 & 95,37 & 99,65 & 119,54 & 119,43 & $p=0.85442$ & $p=0.83746$ & $p=0.0915$ \\
\hline Prominencia labial (labio superior) (**) & 5,86 & 5.56 & 1.93 & 1,96 & 3.93 & 3.87 & 7,79 & 7,25 & $p=0.93014$ & $p=0.90558$ & $p=0.0697$ \\
\hline Prominencia labial (labio inferior) (") & 4.02 & 3.5 & 3.05 & 2.31 & 0.97 & 1.19 & 7,07 & 5.81 & $p=0.66499$ & $p=0.75706$ & $p=0.1341$ \\
\hline Plano Estético (labio inferior) ("*) & 0,88 & 0,4 & 3,39 & 2,76 & $-2,51$ & $-2,36$ & 4,27 & 3,16 & $p=0.72455$ & $p=0.79280$ & $p=0.1722$ \\
\hline
\end{tabular}

\section{DISCUSIÓN}

Mediante el uso de fotogrametría, este estudio buscó determinar el efecto de la TM en el perfil facial de pacientes con maloclusión y disfunción orofacial, con el objetivo de evaluar si esta genera cambios mensurables, entre el inicio y el final del tratamiento fonoaudiológico.

No obstante que no hubo diferencias estadísticas, cabe destacar que se observaron cambios funcionales percibidos por los pacientes y especialistas, tales como cantidad de tiempo que el voluntario logra mantener una respiración nasal v/s oral; manejo de los alimentos de distintas consistencias en la deglución y disminución de los trastornos del habla, los que fueron evaluados por la Fonoaudióloga al finalizar cada sesión de la Terapia.

Visualmente también se observaron cambios positivos en los voluntarios a lo largo de las sesiones de TM, principalmente en la posición de los labios de cada paciente (Figura 4); a pesar de ello, esto no tiene correlato estadístico.
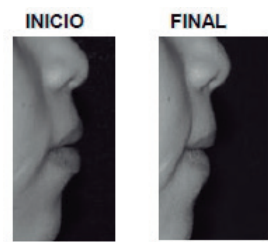

Sujeto 1

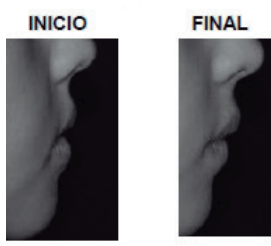

Sujeto 3

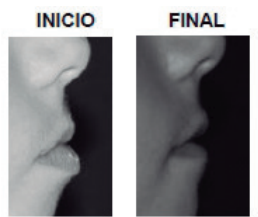

Sujeto 2

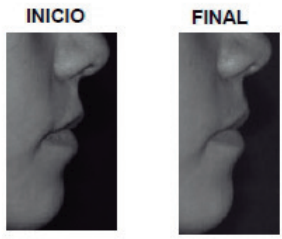

Sujeto 4
Figura 4. Cambios visualmente positivos en voluntarios del estudio. Nótese cambios en posición de los labios al finalizar Terapia Miofuncional.

Respecto a la escasa literatura encontrada sobre el tema, un estudio analizó la relación entre TM y Disfunción Orofacial(14), comprobando que existe un cambio en la actividad electromiográfica de los músculos periorales al finalizar el tratamiento en estos sujetos, lo que podría explicar los cambios visuales observados en los voluntarios. Sin embargo, los cambios funcionales no fueron evaluados en este estudio.

\section{CONCLUSIONES}

Este estudio concluye que la Terapia Miofuncional no tiene efectos mensurables en las mediciones del perfil facial en pacientes con maloclusión y disfunción orofacial, al ser estudiado en fotogrametría.

\section{SUGERENCIAS}

Los cambios funcionales observados no fueron mencionados como resultados debido a que no corresponden a cambios anatómicos o estructurales. Sin embargo, son igualmente relevantes a los resultados expuestos en este estudio debido a que es parte de los objetivos de la TM. Esto cobra importancia ya que esta terapia fue dirigida principalmente a un cambio de función (apuntando a un equilibrio funcional) y no únicamente de morfología. Por esta razón es fundamental poder realizar un diagnóstico ortodóntico riguroso y posterior derivación a Fonoaudiología, si corresponde, para lograr este equilibrio funcional en los pacientes y reducir el riesgo de progresión o reaparición de las 
anomalías Dentomaxilares en el tiempo.

Por último, sería interesante evaluar si existen cambios en los voluntarios después de un año de aplicar y finalizar este tipo de terapias, como también evaluar si existen cambios funcionales a causa de su aplicación, con un tamaño muestral mayor.

Las principales limitaciones de este tipo de estudios es la obtención del tamaño muestral, que es afectada por factores como: la poca adherencia al tratamiento de algunos pacientes y sus apoderados, abandono de tratamiento de algunos de ellos, inasistencias a sesiones agendadas y también no seguir las instrucciones de su tratamiento (como, por ejemplo, realizar ejercicios determinados en casa).

\section{JUSTIFICACIÓN CIENTÍFICA PARA EL ESTUDIO}

Clínicamente se han observado cambios en los tejidos blandos del rostro de los pacientes que reciben Terapia Miofuncional. Esto impacta positivamente en la motivación y satisfacción de estos pacientes y también de los especialistas que realizan este tipo de tratamientos; sin embargo, esta influencia no ha sido estudiada científicamente.

\section{Los resultados principales}

Este estudio demuestra que estos cambios clínicos no tienen un correlato estadístico.

\section{Consecuencias prácticas.}

Es relevante, en la práctica clínica, disponer de esta herramienta para pronosticar los cambios en los pacientes.

\section{CONFLICTO DE INTERÉS}

Los autores no tienen conflicto de interés. Este estudio fue autofinanciado, no recibió financiamiento de ninguna institución ni empresa privada o particulares.

\section{Bibliografía}

1. Borrás S, Rosell V. Capítulo 1: Ortodoncia y terapia miofuncional. En: Borrás S, Rosell V. editores. Guía para la reeducación de la deglución atípica y trastornos asociados. Valencia, España: Nau Llibres; 2005. p. 15-18.

2. Atik E, Akarsu-Guven B, Kocadereli I. Soft tissue effects of three different Class II/1-camouflage treatment strategies. J Orofac Orthop. 2017;78(2):153-65.

3. Janson G, Mendes LM, Junqueira CHZ, Garib DG. Soft-tissue changes in Class II malocclusion patients treated with extractions: a systematic review. Eur J Orthod. 2016;38(6):631-7.

4. Engelke W, Jung K, Knösel M. Intra-oral compartment pressures: A biofunctional model and experimental measurements under different conditions of posture. Clin Oral Investig. 2011;15(2):165-76.

5. Farkas LG, Bryson W, Klotz J. Is photogrammetry of the face reliable? Plast Reconstr Surg.1980;66(3):346-55.

6. Han K, Kwon HJ, Choi TH, Kim JH, Son D. Comparison of anthropometry with photogrammetry based on a standardized clinical photographic technique using a cephalostat and chair. J Cranio-Maxillofacial Surg. 2010;38(2):96-107.

7. Aksu M, Kaya D, Kocadereli I. Reliability of reference distances used in photogrammetry. Angle Orthod. 2010;80(4):670-7.
8. Korb L. Efetividade de um programa de Terapia Miofuncional no o tratamento da respiracao oral em individuos com má-oclusao dentaria: ensaio clinico. [tesis doctoral]. Sao Paulo, Universidad de Sao Paulo, 2017

9. Astudillo-Loyola MP, Dehghan-Manshadi-Kemm S, Vergara-Nuñez C, PeñafielEkdhal C. Son confiables las fotografías para el análisis facial en ortodoncia? Rev Clin Periodoncia Implantol Rehabil Oral. 2018;11(1):13-5.

10. Cuccia AM, Carola C. The measurement of craniocervical posture: A simple method to evaluate head position. Int J Pediatr Otorhinolaryngol. 2009;73(12):1732-6. 11. Solow B, Tallgren A. Natural head position in standing subjects. Acta Odontol Scand. 1971; 29(5):591-607.

12. Legan HL, Burstone CJ. Soft tissue cephalometric analysis for orthognathic surgery. J Oral Surg. 1980;38(10):744-51.

13. Ricketts RM. Bioprogressive therapy as an answer to orthodontic needs. Am J Orthod. 1976;70(03):241-68.

14.Schievano D, Rontani RMP, Bérzin F. Influence of myofunctional therapy on the perioral muscles. Clinical and electromyographic evaluations. J Oral Rehabil. 1999;26(7):564-9. 